\title{
Laser Doppler flowmetry as a measure of extrinsic colonic innervation in functional bowel disease
}

\author{
A V Emmanuel, M A Kamm
}

\begin{abstract}
Background-In functional disorders it is unknown whether disturbed function is due to an intrinsic gut abnormality or altered extrinsic innervation.

Aims-To study whether measurement of mucosal blood flow could be used as a quantitative direct measure of gut extrinsic nerve autonomic activity in patients with idiopathic constipation.

Methods-Seventy two patients with idiopathic constipation and 26 healthy volunteers had rectal mucosal blood flow measurements by a laser Doppler flowmetry probe applied $10 \mathrm{~cm}$ from the anus. Measurements were made at rest and after inhaled placebo and ipratropium $40 \mu \mathrm{g}$.

Results-Constipated subjects had lower baseline rectal blood flow than controls. Patients with slow transit had lower mucosal blood flow than normal transit. The number of retained markers on $x$ ray was inversely correlated with blood flow. Ipratropium reduced blood flow compared with placebo, reduced it less in constipated patients than controls, and reduced it less in patients with slow compared with normal transit. Constipated patients, not controls, showed a significantly attenuated $R R$ interval (the interval between successive $R$ waves on the ECG) variability, and blood flow correlated with vagal function.

Conclusions-Laser Doppler mucosal flowmetry is a gut specific, quantitative measure of extrinsic autonomic nerve activity. The technique has shown that patients with idiopathic constipation have impaired extrinsic gut nerve activity, and this is more notable in those with slow transit. The degree of slow transit correlates with the degree of impaired extrinsic innervation.

(Gut 2000;46:212-217)
\end{abstract}

Keywords: laser Doppler; autonomic nervous system; constipation; mucosal blood flow

Physiology Unit, St Mark's Hospital, Northwick Park, Watford Road, Harrow, Middlesex HA1 3UJ, UK

A V Emmanuel

M A Kamm

Correspondence to: Professor M A Kamm.

Accepted for publication 23 August 1999
Changes in gut motor function can occur because of structural abnormalities of the intrinsic or extrinsic nervous systems innervating the gut. The former includes motility disorders such as achalasia, ${ }^{1}$ chronic idiopathic intestinal pseudo-obstruction, ${ }^{2}$ and Hirschsprung's disease, ${ }^{3}$ while the latter includes the changed gut function observed after pelvic surgery ${ }^{4}$ or in patients with spinal injury. ${ }^{56}$

In contrast to these structural abnormalities it has been harder to characterise the changes in gut function which are thought to occur as a result of changes in the activity of extrinsic nerves. Acute stress has served as a useful model to study this. Since the earliest studies of gastric $^{78}$ and rectal ${ }^{9}$ responses to acute stress in man, it has become clear that acute stress has a direct effect on gut function, changing gut blood flow, motor contractility, and sensitivity. Animal studies have characterised some of the central pathways and the chemical mediators involved these responses ${ }^{10-12}$; their final common pathway is via autonomic extrinsic nerves to the gut rather than neurohumoral mechanisms.

While acute stress can be studied conveniently, chronic stress and other states of chronically disturbed psychological function are more difficult to study. Direct physiological evidence that chronic stress affects the gut has been harder to obtain, because of the difficulty in quantitating levels of chronic stress and because there has been no direct quantitative means of measuring the gastrointestinal effect of that stress. As in acute stress, the effects of chronic stress on the gut are likely to be mediated at least in part via extrinsic nerves to the gut.

Previous studies which have tried to address these questions have utilised indirect autonomic measures, which are thought to correlate with changes in the level of activity of gut innervation. These studies have shown vagal dysfunction in constipation predominant irritable bowel syndrome (IBS), sympathetic adrenergic dysfunction in diarrhoea predominant IBS, ${ }^{13}$ impaired vagal cholinergic activity in patients with idiopathic constipation, ${ }^{14}$ and enhanced parasympathetic activity with diminished sympathetic activity in patients with functional abdominal pain..$^{15} \mathrm{~A}$ direct causative role has not been established between these alterations of autonomic function and the patients' symptoms or changes in gut function.

These existing measures of autonomic activation relate to the cardiovascular system, skin sweating, and circulating hormones. However, their level of activity may bear no relation to the level of gut activation, in view of the specialised pathways from the brain which innervate the different organ systems.

In view of the fact that gastrointestinal mucosal blood flow is thought to be regulated in part by the extrinsic nerve supply, ${ }^{1617}$ we have postulated that assessment of mucosal blood flow may provide a quantitative measure of the level of activity of the nerves innervating

Abbreviations used in this paper: IBS, irritable bowel syndrome; RMBF, rectal mucosal blood flow; RRI, RR interval; VR, Valsalva ratio. 
Table 1 Demographic and symptom breakdown of study subjects

\begin{tabular}{lll}
\hline & Male:female & Mean age (range) \\
\hline Controls $(\mathrm{n}=26)$ & $9: 17$ & $36.5(18-61)$ \\
Patients $(\mathrm{n}=72)$ & $9: 63$ & $40.1(17-69)$ \\
Slow transit $(\mathrm{n}=28)$ & $3: 25$ & $38.0(17-57)$ \\
Normal transit $(\mathrm{n}=44)$ & $6: 38$ & $41.3(18-69)$ \\
\hline
\end{tabular}

the gut. We have used laser Doppler flowmetry to quantitate this.

To determine the possible value of this technique in the assessment of functional gastrointestinal disorders we felt it was necessary to define a group with some objective marker of physiological abnormality, namely slow gut transit. We therefore investigated two groups of patients with a similar subjective complaint (constipation), but with one group having slow, and the other normal gut transit. Having established whether mucosal blood flow differed in either of these conditions from normal, we wished to determine whether assessment of gut mucosal blood flow correlated with conventional autonomic testing. Finally we wished to confirm that changes in the microcirculation in these patients are directly under immediate control of extrinsic autonomic nerves by modifying this nerve activity with inhaled autonomically active drugs.

\section{Methods}

SUBJECTS

Seventy two consecutive adult patients (63 women; mean age 40 years, range 17-69) referred to a tertiary centre with constipation were studied (table 1). All patients complained of either decreased bowel frequency, or unsatisfied defecation with an excessive need to strain. This was not quantitated using a diary system; the subjective complaints were considered adequate for entry to the study in those patients in whom measured transit was normal. All had a normal diameter rectum and colon, and were considered to have idiopathic constipation. Organic and metabolic causes of constipation had been excluded by endoscopy, barium enema, and blood tests. Patients who had taken laxatives within six months of the study or who had taken laxatives regularly for more than six months in total were excluded from the study. Patients taking any regular drug therapy which might interfere with autonomic activity (such as antidepressants, antipsychotics, non-steroidal anti-inflammatory agents, anticonvulsants, antianginals, antihypertensives, or bronchodilators) were excluded from the study.

Twenty six healthy volunteers (17 women; mean age 36 years, range 18-61) were studied as controls.

All women in the study were studied in the follicular phase of the menstrual cycle or were postmenopausal. ${ }^{18}$ No subjects had asthma or chronic lung disease. All patients and volunteers gave informed consent to participate in the study which was approved by the Northwick Park and St Mark's Ethics Committee.
WHOLE GUT TRANSIT

Patients complaining of constipation had their whole gut transit time assessed using radioopaque markers (Dunn Clinical Nutrition, Cambridge, UK). Three sets of 20 markers which were radiologically distinct were ingested at 24 hour intervals on three consecutive days and a plain abdominal $x$ ray was obtained 120 hours after ingestion of the first set of markers. ${ }^{19}$ A patient was deemed to have slow transit if there was retention of more than the normal for any one of the three sets of markers according to our previously defined and published normal range. ${ }^{19}$ The remaining patients were classified as having "normal transit constipation".

\section{LASER DOPPLER BLOOD FLOW}

Laser Doppler flowmetry measures changes in red cell flux. However, using a probe which scans a fixed volume of tissues, an indirect measure of flow can be obtained. The methodology and reproducibility of this technique have been published previously. ${ }^{18}$ Subjects were studied in a room maintained at $22^{\circ} \mathrm{C}$ after 15 minutes acclimatisation. Subjects were studied in the left lateral position. After digital examination and rigid sigmoidoscopy had confirmed the rectum to be empty the laser Doppler probe was introduced via the sigmoidoscope. Recordings were made at $10 \mathrm{~cm}$ from the anal verge at four points circumferentially at 90 degrees to each other. Readings were taken after the trace had stabilised for 30 seconds.

The coefficient of variability of laser Doppler flowmetry has been shown to be less than $10 \%$ when subjects are studied under identical conditions of menstrual phase and fasting. We have previously shown that measurements are optimally made by averaging four circumferential sites, thus minimising any regional variation in flux values. ${ }^{18}$ Performing the measurements was entirely painless for all subjects and there was no change in flux values even over prolonged periods of measurement.

To compare simultaneously the blood flow in the same sacral visceral and somatic innervations the skin blood flow over the S2 dermatome was also determined.

\section{BRONCHODILATOR INHALATION}

To assess the effect on extrinsic gut nerve activity of acutely applied autonomic drugs, 42 patients (38 women, 19 slow transit) and 26 healthy controls underwent further studies with inhaled autonomically active bronchodilators. All subjects were studied on two separate occasions, with two test inhalations on each occasion. On each occasion this consisted of placebo and either the muscarinic antagonist ipratropium $40 \mu \mathrm{g}$ or the $\beta_{2}$ agonist salbutamol $200 \mu \mathrm{g}$, in random order, separated by $60 \mathrm{~min}-$ utes. These doses were used as they were sufficient to raise the heart rate by greater than $10 \%$; induction of a tachycardia was used as an indicator of systemic availability of the drug. Inhalation was administered using a spacing device. The studies were conducted in a double blind manner, using unlabelled inhalers. We 
have previously shown that sympathetic $\beta_{1}$ blockade reduces mucosal blood flow, confirming that the major inhibitory influence on gut motor function ${ }^{20}$ and blood flow is sympathetic. A muscarinic antagonist was used to determine whether autonomic sacral efferent cholinergic nerves influence mucosal blood flow. A $\beta_{2}$ agonist was used to test whether mucosal sympathetic vasodilator tone could be increased.

\section{CARDIORESPIRATORY AUTONOMIC FUNCTION} TESTS

Vagal cholinergic function was measured using two methods. The interval between successive $R$ waves on the electrocardiogram ( $R R$ interval) in response to vagovagal reflex bradycardia induced by deep breathing was measured. Secondly, the peak to trough heart rate ratio during a Valsalva manoeuvre, that is the "Valsalva ratio" (VR), was measured. This manoeuvre involves forced expiration against a closed glottis, effectively raising intraabdominal pressure and reducing venous return to the heart.

Sympathetic adrenergic activity was assessed by the percentage change in resting pulse and systolic blood pressure in response to orthostatic tilt of the bed from horizontal to an angle of 30 degrees. Secondly, the systolic blood pressure in phase II (peak systolic blood pressure) and phase IV (trough systolic blood pressure) of the Valsalva manoeuvre were assessed and the "II:IV" ratio determined.

All electrocardiographic data were obtained simultaneously with the laser flowmetry measurement of rectal microcirculation.

Results of cardiorespiratory autonomic testing in patients were compared with the results from the 26 healthy controls and with published normal data. ${ }^{21}$

STATISTICAL ANALYSIS

Data were normally distributed, and are therefore expressed as mean (SEM). The two tailed $t$ test was used to compare the results of rectal mucosal blood flow and autonomic cardiorespiratory function tests between the two patient groups and controls. Spearman's rank order correlation (with a Bonferroni correction factor of 0.01 to adjust for multiple variables) was used to determine associations between the amount of retained markers in patients with constipation, laser "flux" values, and cardiorespiratory autonomic test results. Results were significant at $\mathrm{p}<0.05$.

\section{Results}

WHOLE GUT TRANSIT

Twenty eight patients ( 25 women; mean age 38 years, range 17-57) had slow transit and 44 (38 women, mean age 41 years, range 18-69) had normal transit (table 1 ).

LASER DOPPLER BLOOD FLOW

Rectum

Baseline blood flow in the rectal mucosa was significantly lower in patients compared with controls (156.5 (14.2) versus 186.0 (14.2), $\mathrm{p}=0.026$; fig 1 ). Patients with slow transit had

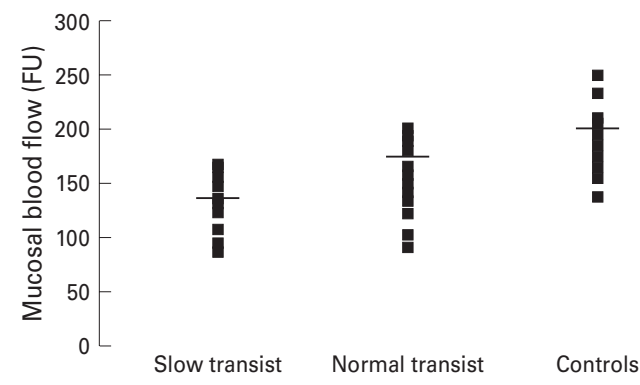

Figure 1 Rectal mucosal blood flow in patients and controls. Flow is lowest in patients with slow compared with normal transit $(p=0.044)$ and compared with healthy controls ( $p=0.015)$; mean bars are shown. Note relatively tighter distribution of blood flow values in slow transit patients.

significantly lower mucosal blood flow than those with normal transit (145.8 (15.9) versus 167.0 (13.3), $\mathrm{p}=0.044$ ).

Skin

Sacral dermal blood flow was similar in both groups of subjects (118.5 (13.3) versus 126.3 (11.7), patients versus controls, $\mathrm{p}=0.45)$. There was also no significant difference in skin blood flow between patients with slow transit and patients with normal transit.

EFFECT OF INHALED BRONCHODILATORS ON MUCOSAL BLOOD FLOW

Inhaled ipratropium at a dose of $40 \mu \mathrm{g}$ raised heart rate in $42 \%$ of patients and 25 of 26 control subjects by over $10 \%$ (mean $11.1 \%$ ); inhaled salbutamol $200 \mu \mathrm{g}$ increased heart rate by over $10 \%$ in 39 of 42 patients and 25 of 26 controls (mean 11.3\%).

Gut

Patients with constipation had a significant reduction in mucosal blood flow in response to inhaled ipratropium compared with placebo (mean reduction $10.3 \%$ versus $4.3 \%$, ipratropium versus placebo, $p=0.008$; fig 2 ). The patients with slow transit had a significantly smaller reduction response to ipratropium than those with normal transit $(6.8 \%$ versus $14.2 \%$, $\mathrm{p}=0.030)$. The response of slow transit patients to ipratropium did not differ significantly from the response to placebo $(6.8 \%$ versus $4.3 \%$, $\mathrm{p}=0.22$ ).

Control subjects showed a significant reduction in mucosal blood flow in response to inhaled ipratropium compared with placebo (mean reduction $15.7 \%$ versus $2.5 \%$, ipratropium versus placebo, $\mathrm{p}=0.002$ ).

The mean reduction in flow in response to inhaled ipratropium was significantly less in constipated patients compared with controls $(10.2 \%$ versus $15.7 \%, \mathrm{p}=0.040)$

Inhaled salbutamol did not significantly affect rectal mucosal blood flow in constipated patients $(0.2 \%$ increase versus $3.1 \%$ reduction, salbutamol versus placebo, $\mathrm{p}=0.11$ ) or controls (mean increase $2.3 \%$ versus mean increase $0.2 \%, \mathrm{p}=0.20)$.

Skin

In both patients and controls sacral dermal blood flow was not significantly affected by inhaled ipratropium (placebo versus 


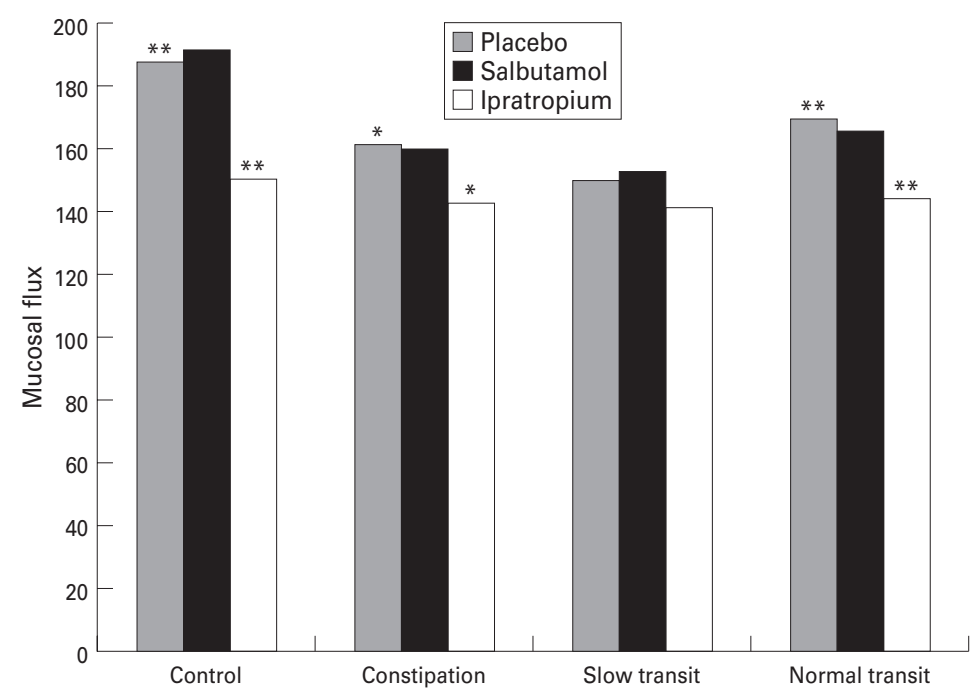

Figure 2 Rectal mucosal blood flow in response to inhaled placebo, salbutamol $200 \mu \mathrm{g}$, and ipratropium $40 \mu \mathrm{g}$. Ipratropium, and not salbutamol, caused a reduction in blood flow in controls and in patients with normal transit constipation, but not in those with slow transit constipation. ${ }^{\star} p<0.05,{ }^{\star *} p<0.01$.

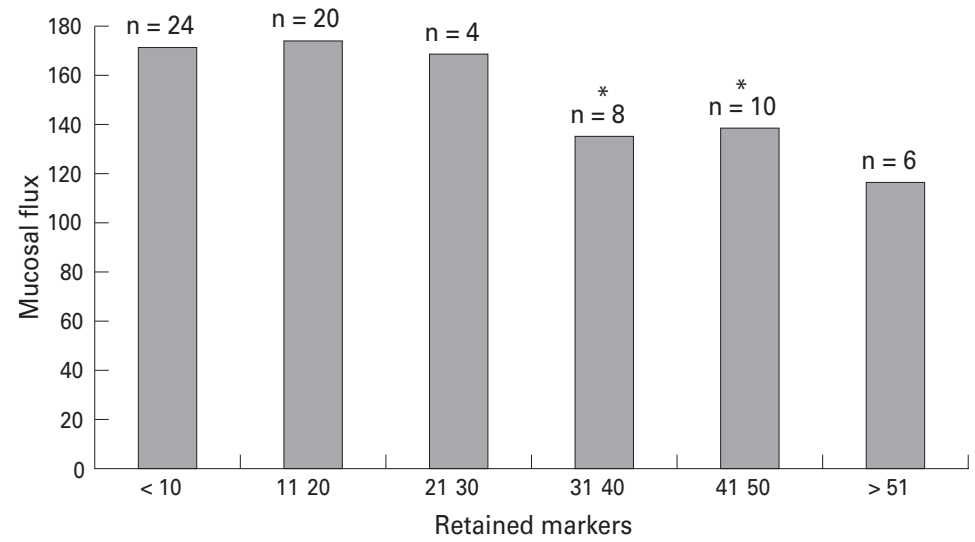

Figure 3 Relation between rectal mucosal blood flow and radio-opaque markers retained on $x$ ray in transit study. There was a strong inverse correlation between blood flow and number of retained markers (slower transit). ${ }^{*} p<0.05, t p<0.03$.

Table 2 Cardiorespiratory autonomic function testing in constipated patients and controls

\begin{tabular}{lllll}
\hline & RR variability & Valsalva ratio & $\begin{array}{l}\text { Orthostatic } \\
\text { adjustment ratio }\end{array}$ & $\begin{array}{l}\text { Phase II:IV } \\
\text { ratio }\end{array}$ \\
\hline Controls $(\mathrm{n}=26)$ & 24.5 & 1.62 & -3.9 & 1.64 \\
Constipation $(\mathrm{n}=72)$ & $11.2^{\star \star \star}$ & $1.16^{\star}$ & $-4.8 \ddagger$ & $1.44 \ddagger$ \\
Slow transit $(\mathrm{n}=28)$ & $7.5^{\star \star}$ & $1.12 \dagger$ & $4.4 \ddagger$ & $1.53 \ddagger$ \\
Normal transit $(\mathrm{n}=44)$ & $13.6^{\star \star \star}$ & $1.18^{\star}$ & $5.0 \ddagger$ & $1.38 \ddagger$ \\
\hline
\end{tabular}

${ }^{\star \star \star} \mathrm{p}<0.001 ;{ }^{\star \star} \mathrm{p}<0.01 ;{ }^{\star} \mathrm{p}<0.05 ; \mathrm{tp}<0.07 ; \ddagger \mathrm{NS}$.

ipratropium: 116.5 versus 122.7 patients; 121.4 versus 118.7 controls; $\mathrm{p}=0.115$ and 0.134 respectively) or salbutamol (placebo versus salbutamol: 123.6 versus $117.7 \mathrm{pa}-$ tients; 111.9 versus 125.6 controls; $\mathrm{p}=0.108$ and 0.121 respectively).

RELATION BETWEEN RECTAL MUCOSAL BLOOD FLOW AND WHOLE GUT TRANSIT

There was a significant and strong inverse correlation between rectal mucosal blood flow and the total number of retained markers on abdominal $x$ ray $(r=-0.87, \mathrm{p}=0.026$; fig 3 ). When the patient group was divided into those with 30-40, 40-50, or 50-60 retained markers, those patients with greater retained markers had significantly lower rectal mucosal blood flow (RMBF) compared with those patients with $0-10,10-20$, and $20-30$ retained markers, when each was tested separately. The upper limit of normal for total number of retained markers in our laboratory, derived from a series of 25 men and 18 women, is 19 (out of a total of 60$)$ markers. ${ }^{19}$

RELATION BETWEEN MUCOSAL BLOOD FLOW AND AUTONOMIC FUNCTION

When considering all 98 subjects in this study (constipated patients and healthy controls), there was a significant correlation between RR interval (RRI) variability and rectal flux $(t=-4.4, \mathrm{p}=0.0042$; table 2, fig 4$)$ and between VR and rectal flux $(t=-3.7, \mathrm{p}=0.027)$.

There were no differences in the adrenergic measures (orthostatic adjustment ratio and phase II:IV VR) between the controls and patients with constipation. However, the vagal cholinergic measures of RRI variability $(t=-3.2, \mathrm{p}=0.006)$ and VR $(t=-2.8, \mathrm{p}=0.033)$ were significantly different for the constipated patients compared with controls.

There was also a significant correlation between these measures of cholinergic function and the rectal blood flow measurement in the patients with slow transit (RRI: $t=-5.7$, $\mathrm{p}=0.001$; VR: $t=-3.6, \mathrm{p}=0.032$ ). These parameters did not correlate significantly in normal transit patients or controls.

\section{RELATION BETWEEN AUTONOMIC FUNCTION AND} TRANSIT

When considering all 72 patients complaining of constipation there was a significant correlation between the vagal cholinergic scores and number of retained markers (RRI: $t=-4.8$, $\mathrm{p}=0.0040$; VR: $t=-3.8, \mathrm{p}=0.019)$. The retention of greater than 30 of the original 60 markers was associated with the more abnormal vagal autonomic scores and rectal blood flow. Using multivariant statistical analysis there was a significant correlation between the vagal cholinergic RRI test score and rectal blood flow when considering all 72 constipated patients $(r=0.92, \mathrm{p}=0.011)$.

\section{Discussion}

We have shown that patients with constipation have evidence of vagal cholinergic dysfunction, as illustrated both by conventional cardiorespiratory autonomic tests and by the novel gut specific measure of rectal mucosal blood flow measurement. These findings support those previous studies in which peripheral autonomic testing was applied. ${ }^{1322} 23$ This cholinergic abnormality was most profound in those with slow transit constipation. The cholinergic output to the gut is via vagal and sacral efferents. This study and previous studies $^{1322} 23$ suggest that in patients with constipation there are altered cardiovascular and sacral efferents. Vagal cholinergic activity to the gut has not been tested.

A potential limitation of the interpretation of conventional autonomic function tests in functional gut illnesses is that they assume that cardiorespiratory dysfunction mirrors gastrointestinal dysfunction. In patients with 
polyneuropathy ${ }^{24}$ there is known to be poor correlation between serum pancreatic polypeptide assays and electrocardiographic assessments of autonomic function. Similarly, in functional bowel disorders ${ }^{25}$ there is a variable relation between measurement of plasma catecholamines and cardiorespiratory sympathetic tests. In contrast, in a previous study of healthy volunteers we have shown that the measurement of rectal mucosal blood flow is a sensitive and reproducible index of intraabdominal autonomic activity. ${ }^{18}$

We have provided the first report of altered resting rectal mucosal blood flow in patients with constipation. This reduction in blood flow is most notable in those with slow transit, suggesting that these patients form a distinct subgroup of the population of constipated patients, with distinct physiological differences from those with normal transit. We have also shown that the reduction in microcirculatory flow is strongly correlated with the parasympathetic cholinergic dysfunction, as evidenced by altered cardiovascular reflexes. This would support the use of laser Doppler flowmetry as an index of the level of activity of extrinsic innervation of the colon. The use of blood flow measures as an index of autonomic activity is not without precedent. Chaudhuri and colleagues ${ }^{26}$ have shown a correlation between superior mesenteric artery blood flow and intra-abdominal autonomic activity, and Thomaides and colleagues ${ }^{27}$ have shown that such measurements are dynamic and change in response to autonomically active drugs.

This minimally invasive technique is safe, and simpler than conventional measures of intra-abdominal autonomic activity such as measurement of plasma levels of pancreatic polypeptide in response to hypoglycaemia or sham feeding. ${ }^{24}$ We have previously shown it to be highly reproducible and well tolerated. ${ }^{18}$

In addition to rectal mucosal flux correlating with cardiorespiratory tests of autonomic activity it also correlated strongly with whole gut transit as measured using radio-opaque markers. The slower the transit the more profound was the reduction of mucosal flow. This provides strong evidence for the presence of either a strong sympathetic inhibitory drive affecting both colonic transit and mucosal blood flow, or a deficit in the normal cholinergic drive to both these physiological functions in patients with slow transit. The effect of the sympathetic system is inhibitory, ${ }^{20}$ with extra sympathetic drive from bronchodilators having little additional effect. The tests involving inhaled ipratropium provide evidence in support of the sensitivity of rectal flow as a measure of the cholinergic drive. There is a positive cholinergic sacral efferent drive, not just to motor function, ${ }^{28}{ }^{29}$ but also to blood flow. In constipation, especially slow transit, there may already be some diminution in sacral activity, or there is less susceptibility to the inhalers, as evidenced by the lesser blood flow response to cholinergic blockade.

White and colleagues ${ }^{30}$ have shown that patients with abdominal pain in IBS have heightened airway responsiveness to inhaled metacholine, a muscarinic agonist, and these patients have also been to shown to have increased vagal cholinergic activity. ${ }^{15}$ In this study patients with slow transit showed an impaired microcirculation vasodilatory response to a muscarinic receptor antagonist, giving further weight to the concept of reduced cholinergic neural activity in slow transit constipation.

Mucosal blood flow in patients with normal transit differed significantly from controls and those with slow transit. It therefore seems likely that they also have disturbed autonomic innervation, but to a lesser or different degree than in patients with slow transit. We believe changes in blood flow and transit are both results of altered extrinsic innervation. The changes in blood flow should not be thought of as secondary to the altered motility; changes in motility, blood flow, and secretion are all likely to occur simultaneously as is seen in acute experiments. $^{8}{ }^{9}$

We believe that the changes and abnormalities present reflect changes in extrinsic nerve activity. Further evidence for this comes from treatment of such patients with biofeedback, with impaired transit correlating with impaired mucosal blood flow. ${ }^{31}$ Such changes would be unlikely if the primary abnormality were a permanent disorder of intrinsic neural activity. Furthermore, electrical spinal stimulation actively changes mucosal blood flow. ${ }^{32}$ However, we cannot exclude the involvement of other factors which contribute to the regulation of mucosal blood flow, such as intrinsic neurones, local autocrine factors (for example, prostaglandins and nitric oxide), circulating hormones, or some blood vessel abnormality in patients with constipation.

In a separate study ${ }^{33}$ we have shown that patients with constipation have altered psychological profiles to healthy controls. In particular they have greater levels of anxiety, depression, somatisation, and social dysfunction. The only difference we observed between those with slow and normal transit constipation was in respect to somatisation, such that those with normal transit had lower levels of somatisation than those with slow transit. These psychological changes correlate with alterations in blood flow. It therefore appears that there is a strong relation between chronic psychological state, autonomic dysfunction, increased transit time, and altered blood flow. The causal nature of this relation remains to be determined.

In summary, we have shown that idiopathic constipation is associated with a deficit of efferent vagal cholinergic activity. This is more notable in patients with slow transit. Laser Doppler flowmetry offers a reproducible and valid means of studying the level of activity of the extrinsic innervation of the gut in patients with functional disorders.

We are grateful to Dr C Dore for statistical advice, and to Dr A Roy for practical and technical help in performing this work.

1 Friesen DL, Henderson RD, Hanna W. Ultrastructure of the esophageal muscle in achalasia and diffuse oesophageal spasm. Am f Clin Pathol 1983;79:319-23. 
2 Krishnamurthy S, Schuffler M. Pathology of neuromuscular disorders of the small intestine and colon. Gastroenterology 1987;93:610-16.

3 Tam PKH. An immunochemical study with neuro-specificenolase and substance $\mathrm{P}$ of human enteric innervation. The normal developmental pattern and abnormal deviations in Hirschsprung's disease and pyloric stenosis. F Paediatr Surg 1986;21:227-36

4 Gunterberg B, Kewenter J, Petersen I, et al. Anorectal function after major resections of the sacrum with bilateral or unilateral sacrifice of sacral nerves. Br f Surg 1976;63:54654.

5 Connell AM, Frankel H, Guttmann L. The motility of the pelvic colon following complete lesions of the spinal cord Paraplegia 1963;1:98-115.

6 Longo WE, Ballantyne GH, Modlin IM. The colon, anorectum and spinal cord patient: a review of the functional alterations of the denervated hindgut. Dis Colon Rectum 1989;32:261-7.

7 Beaumont W. Experiments and observations on the gastric juice and the pathophysiology of digestion. Plattsburg: FP juice and the pan, 1833 .

8 Wolf S, Wolf HG. Experimental study of changes in gastric function in response to varying life experiences. Rev Gastroenterol 1947;14:419-26.

9 Almy TP. Experimental studies on the irritable colon. $A m \mathcal{F}$ Med 1951;10:60-7.

10 Bueno L, Ferre JP. Central regulation of intestinal motility by somatostatin and cholecystokinin octapeptide. Science 1982;216:1427-9.

11 Bueno L, Gue M, Delrio C. CNS vasopressin mediates emotional stress and $\mathrm{CRH}$-induced colonic motor alterations in rats. Am F Physiol 1992;262:G427-31.

12 Corticotropin-releasing factor and the brain-gut motor response to stress. Can $\mathcal{F}$ Gastroenterol 1999;13(suppl A): $18-25 \mathrm{~A}$

13 Aggarwal A, Cutts TF, Abell TL, et al. Predominant symptoms in irritable bowel syndrome correlate with specific toms in irritable bowel syndrome correlate with specific 1994;106:945-50.

14 Altomare D, Pilot MA, Scott M, et al. Detection of a subclinical autonomic neuropathy in constipated patients using a sweat test. Gut 1992:33:1539-43.

15 Jorgensen LS, Christiansen P, Raundahl U, et al. Autonomic nervous system function in patients with functional abdominal pain. An experimental study. Scand $\mathcal{F}$ Gastroenterol 1993;28:63-8.

16 Jodal M, Lundgren O. Neurohormonal control of gastrointestinal blood flow. In: Schultz SG, Wood JD, Rauner BB, eds. Handbook of physiology, Section 6: the gastrointestinal sys-
tem. Motility and circulation. Bethesda: American Physiological Society, 1989:1667-711.
17 Burnstock G. Integration of factors controlling vascular tone - an overview. Anaesthesiology 1993;79:1368-80.

18 Emmanuel AV, Kamm MA. Laser Doppler measurement of rectal mucosal blood flow. Gut 1999;45:64-9.

19 Evans RC, Kamm MA, Hinton JM, et al. The normal range and a simple diagram for recording whole gut transit time. Int $\mathcal{F}$ Colorectal Dis 1992;7:15-17.

20 McIntyre AS, Thompson DG. Adrenergic control of motor and secretory function in the gastrointestinal tract. Aliment Pharmacol Ther 1992;6:125-42.

21 Ewing DJ. Cardiovascular reflexes and autonomic neuropathy. Clin Sci Med 1978;55:321-7.

22 Smart HL, Atkinson M. Abnormal vagal function in irritable bowel syndrome. Lancet 1987;ii:475-8.

23 Fukudo S, Nomura T, Muranaka M, et al. Brain-gut response to stress and cholinergic stimulation in irritab bowel syndrome. f Clin Gastroenterol 1993;17:133-41.

24 Polinsky RJ, Taylor IL, Chew P, et al. Pancreatic polypeptide responses to hypoglycaemia in chronic autonomic failure. $\mathcal{F}$ Clin Endocrinol Metab 1982;54:48-52.

25 Camilleri M, Balm RK, Low PA. Autonomic dysfunction in patients with chronic intestinal pseudo-obstruction. Clin Auton Res 1993;3:95-100.

26 Chaudhuri KR, Thomaides T, Mathias CJ. Abnormality of superior mesenteric blood flow in human sympathetic failure. F Physiol (Lond) 1992;457:477-89.

27 Thomaides TN, Chaudhuri KR, Maule S, et al. Differential responses in superior mesenteric artery blood flow may explain the variant pressor responses to clonidine in two groups with sympathetic denervation. Clin Sci 1992;83:5964 .

28 Devroede G, Arhan P, Duguay C, et al. Traumatic constipation. Gastroenterology 1979;77:1258-67.

29 MacDonagh R, Sun WM, Thomas DG, et al. Anorectal function in patients with complete supraconal spinal cord lesions. Gut 1992;33:1532-8.

30 White AM, Stevens WH, Upton AR, et al. Airway responsiveness to inhaled methacholine in patients with irritable bowel syndrome. Gastroenterology 1991;100:6874.

31 Emmanuel AV, Kamm MA. Successful response to biofeedback for constipation is associated with specifically improved extrinsic autonomic innervation to the large bowel [abstract]. Gastroenterology 1997;112(suppl):A729.

32 Vaizey CJ, Kamm MA, Turner IC, et al. Effects of short term sacral nerve stimulation on anal and rectal function in patients with anal incontinence. Gut 1999;44:407-12.

33 Emmanuel AV, Kamm MA, Mason HJ. Direct evidence of psychological state affecting extrinsic innervation of the gut-laser Doppler flowmetry to measure mucosal blood flow [abstract]. Gastroenterology 1997;112(suppl):A728. 